\title{
Galactic kinematics near the sun from open clusters $\dagger$
}

\author{
M. Shen \\ Department of Astronomy, Nanjing University, China \\ email: shenming@nju.org.cn
}

\begin{abstract}
A catalogue of open clusters is used to analyze the Galactic kinematics near the Sun. The Galactic open clusters, which are components of Galactic thin disk, were selected for our analysis. Based on kinematical data for around 270 Galactic open clusters, we found the Galactic rotation curve remains flat near the Sun. We also found $V_{e}=6.40 \pm 3.39 \mathrm{~km} \mathrm{~s}^{-1}$ inside the solar circle, which shows a weak trend of stars moving toward the Galactic anti-center.
\end{abstract}

Keywords. Galaxy: kinematics and dynamics, open clusters and associations: general

\section{Observation data}

Kharchenko et al. (2005) identified 520 Galactic open clusters and compact associations on the basis of the All-Sky Compiled Catalogue which contains 2.5 million stars (ASCC2.5) (Kharchenko 2001). Dias et al. (2002, 2004) collected all published open clusters from the available literature, resulting in 1637 clusters in their DAML02 catalogue.

In this work, we used the data of 520 open clusters from Kharchenko et al. (2005), in which 253 open clusters have heliocentric distances, mean proper motions, mean radial velocities, and ages. For the remaining clusters, 29 mean radial velocity measurements are taken from the DAML02. In addition, another 19 clusters with complete kinematical data in DAML02 were also used. We found spatial velocities for 301 clusters from our sample, in which 10 clusters were excluded because of their suspect peculiar velocity.

Figure 1 displays 137 clusters younger than $50 \mathrm{Myr}$, projected on the Galactic plane and clearly showing 3 arms. The clusters older that than $120 \mathrm{Myr}$ do not match well the location of spiral arms, and thus were not used in this study.

\section{Models \& results}

Consider a rotating expanding ring around the Galactic center, in which the velocity of an arbitrary point can be written as $\Omega \times \mathbf{R}+\left(v_{e} / R\right) \mathbf{R}$, where $\Omega$ is the angular velocity, $\mathbf{R}$ is the Galactocentric position vector of the point, and $v_{e}$ stands for the velocity to the Galactic center. When observed from the Sun, the line-of-sight velocity can be written as follows,

$$
v_{l o s}(l)=\left(\left[\Omega(R)-\Omega\left(R_{0}\right)\right] R_{0} \sin \ell+\frac{d^{2}+R^{2}-R_{0}^{2}}{2 R d} v_{e}-u_{0} \cos \ell-v_{0} \sin \ell\right) \cos b-w_{0} \sin b,
$$

In this equation, $\Omega\left(R_{0}\right)$ is angular velocity at the Sun, and $d$ is the heliocentric distance to the point in the ring.

When analyzing Hipparcos main sequence stars, Dehnen \& Binney (1998) found

$\dagger$ Supported by the National Natural Science Foundation of China(Grant Nos. 10333050 and $10673005)$ 


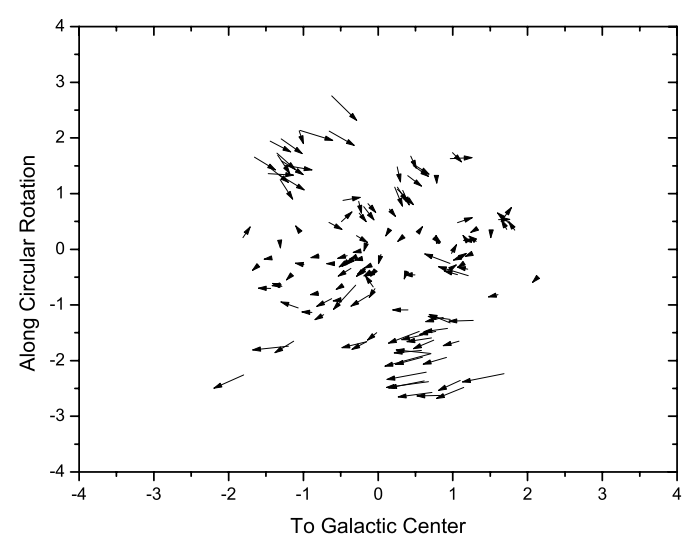

Figure 1. Distribution of 137 young open clusters in the Galactic plane. The vectors show the peculiar motion of clusters.

$u_{0}=10.0 \pm 0.4 \mathrm{~km} \mathrm{~s}^{-1}, v_{0}=5.2 \pm 0.6 \mathrm{~km} \mathrm{~s}^{-1}$, and $w_{0}=7.2 \pm 0.4 \mathrm{~km} \mathrm{~s}^{-1}$ for the solar motion. In the following analysis, $u_{0}, v_{0}, w_{0}$ are fixed.

The Galactocentric radius $R$ of a star is given by

$$
R=\left(R_{0}^{2}+d^{2} \cos ^{2} b-2 R_{0} d \cos \ell \cos b\right)^{\frac{1}{2}} .
$$

As mentioned, 137 young open clusters are distribute over the three arms. A few clusters belong to the outer arm, thus we mainly studied the inner and the local arm. We adopted a simple model, in which by changing the arm's radius we tried to minimize the mean distance of our clusters to that arm. The clusters farther than $0.5 \mathrm{kpc}$ from the arm were not used in our analysis.

Equations (2.1) for radial velocities are to be solved via least squares. Then we have,

$$
\begin{aligned}
\Omega(R)-\Omega\left(R_{0}\right) & =0.67 \pm 0.35 \mathrm{~km} \mathrm{~s}^{-1} \mathrm{kpc}^{-1} \\
v_{e} & =6.40 \pm 3.39 \mathrm{~km} \mathrm{~s}^{-1} \text { for inner arm and } \\
\Omega(R)-\Omega\left(R_{0}\right) & =-2.16 \pm 0.59 \mathrm{~km} \mathrm{~s}^{-1} \mathrm{kpc}^{-1} \\
v_{e} & =3.68 \pm 5.78 \mathrm{~km} \mathrm{~s}^{-1} \text { for local arm. }
\end{aligned}
$$

This result shows a flat rotation curve near the Sun, and a weak trend of stars moving toward the Galactic anti-center. Due to the large error in $v_{e}$, this trend is not definite.

\section{References}

Dehnen, W. \& Binney, J. 1998, MNRAS, 298, 387

Dias, W. S., Alessi, B. S., Motinho, A., \& Lépine J. R. D 2002, A\& $A, 389,871$

Dias, W. S, Lépine, J. R. D, Alessi, B. S., \& Moitinho, A. 2004, Catalog of Optically Visible

Open Clusters and Candidates (DAML02), http://www.astro.iag.usp.br/ wilton/

Kharchenko, N. V. 2001, Kinematics and Physics of Celestial Bodies, 17, 409

Kharchenko, N. V., Piskunov, A. E., Röser S., et al., 2005, A $\& A$ A, 438, 1163 